\section{Periodic 3D Structures Prepared by Photolithography}

Methods for large-scale production of micron-sized periodic structures are of great interest for the manufacture of photonic crystals, membranes, data-storage materials, and catalyst supports. Recently, Shu Yang and co-workers at Bell Laboratories, Lucent Technologies and Princeton University have demonstrated a photolithographic method for producing organic epoxy structures in an area larger than $1 \mathrm{~mm}$ in diameter. The structures have hexagonal, square, or fcc symmetries with periods of $0.9-8 \mu \mathrm{m}$. The symmetry and periodicity of the structures were templated by the interference pattern of continuous-wave $(\mathrm{CW})$ laser radiation in the visible wavelength range. According to Yang, "the use of CW visible laser radiation results in a manufacturing speed and structure size advantages over two-photon infra red and pulsed ultraviolet interference photolithographic methods, respectively."

As described in the July issue of Chemistry of Materials, the researchers controlled the acid catalyzed polymerization of the Epon SU-8 epoxy monomer through the use of 2,4,5,7-tetraiodo-6-hydroxy-3-fluorene as the photosensitizing dye, diaryliodionium hexaflouroantimonate as the photoacid generator, and triethylamine (TEA) as the photoacid neutralizer. These reagents were mixed in tetrahydrofuran and spin-cast onto a cover glass to produce a solid 4-10- $\mu$ m-thick resist film. The researchers initiated polymerization by irradiating the resist for $1 \mathrm{~s}$ with three or four non-coplanar beams of 514-nm Ar ion laser radiation to produce localized photoacids, and polymerization was chemically amplified when heating at $65^{\circ} \mathrm{C}$. The triethylamine played a key role in the resist to neutralize the background photoacids and to increase the sharpness of the structure features. Following polymerization, the structure was washed with propylene glycol methyl ether acetate to remove the unexposed film, and dried in supercritical $\mathrm{CO}_{2}$. Scanning electron microscopy was used to confirm the formation of the highly periodic structure of the specified size and symmetry.

The use of visible laser radiation in the photolithographic process resulted in higher substrate penetration efficiency and more even intensity distribution than UV radiation. This allowed for the production of larger structures and structures with fewer defects.

The number of defects was further reduced by the inherent stability of $\mathrm{CW}$ radiation. Visible radiation also allows CW lithography to be used on a wider range of substrates than does UV radia- tion. The researchers were able to alter lattice constants, porosities, and symmetry of the lattice by varying TEA concentrations, exposure times, laser intensities, beam orientations, and beam polarizations, and by using a laser mask.

GREG KHITROV

\section{Polymer Molds Replicate \\ Micropatterns to Ceramic Surfaces}

Micropatterning of ceramic surfaces is becoming increasingly important in ceramic manufacturing. Micropatterned components make it possible to integrate more entities into a small area and the smaller size decreases the probability of failure for ceramic components. Micropatterned parts can be beneficial in fuel cells to increase the active surface area of dense components or for the fabrication of microchannels for effective gas distribution. Moreover, micropatterning enables the assembly of ceramic components into microelectromechanical systems (MEMS) fabricated with silicon-based technologies.

Using nonporous polymer molds, Urs Schönhozer and co-workers at the Swiss Federal Institute of Technology (ETH Zurich) successfully patterned ceramic surfaces with a feature resolution in the micrometer range. High-quality line patterns with an aspect ratio of 1 and a pitch of $3 \mu \mathrm{m}$ were replicated. The details were described in an article published in the July issue of the Journal of the American Ceramic Society.

In the first experiment, the feasibility of replication was evaluated with powders of zirconia, cerium gadolinium oxide (CGO), and tin oxide. The researchers first cast poly(dimethylsiloxane) (PDMS) onto a $3 \times 3 \mathrm{~mm}^{2}$ master template of etched silicon grating. After curing, the PDMS molds were peeled off the master template. Suspensions of the ceramic powders (all with an average particle size of $<0.3 \mu \mathrm{m}$ ) were cast onto the PDMS mold. After drying and sintering, $80 \%$ of the patterned ceramic surface area was defectfree. The pattern geometry was clearly resolved in all three sintered parts. No wear was observed on the master template following several production cycles.

In the second experiment, the researchers compared the pattern formation of alumina on various mold materials. They concluded that hydrophobic mold surfaces such as PDMS, high-density polyethylene (HDPE), and poly(tetrafluoroethylene-cohexafluoropropylene resulted in excellent and almost identical pattern quality, while hydrophilic mode surfaces, such as plasma-treated PDMS and epoxy, caused a relatively poor pattern quality.

SHIMING Wu

\section{DNA Serves as Effective Metallization Template}

The potential for DNA as a metallization template has been demonstrated by researchers from Technische Universität Dresden and the Università di Trieste, Italy. As reported in the August issue of Nano Letters, a research team led by Michael Mertig of Dresden found that $\mathrm{Pt}(\mathrm{II}) \cdot$ DNA adducts, which are complexes formed during incubation of DNA in a tetrachloroplatinate solution, may act as extremely efficient nucleation centers for metal cluster formation. Therefore, the balance between heterogeneous metal cluster nucleation on the biomolecular template and unwanted homogeneous nucleation of clusters in bulk solution can be controlled in a way that achieves selectively heterogeneous metallization. As a result, platinum cluster necklaces of unprecedented thinness and uniformity were fabricated with purely heterogeneous metallic growth on DNA. This biomolecular template-based technology represents a promising route for production of complex metal nanostructures.

The Dresden/Trieste group began the investigation with first-principles moleculardynamics (FPMD) simulations to elucidate the molecular mechanism of platinum cluster nucleation on DNA templates. Using guanine $(G)$ to represent a generic DNA base containing a nitrogen binding site, the $\mathrm{Pt}(\mathrm{II}) \cdot \mathrm{DNA}$ adducts were modeled as either a partially hydrolyzed complex bound to guanine, $\mathrm{G} \cdot \mathrm{PtCl}_{2}-\left(\mathrm{H}_{2} \mathrm{O}\right)[\mathrm{GP}]$ or by a fully hydrolyzed complex bound to two stacked guanines, $\mathrm{GG} \cdot \mathrm{Pt}\left(\mathrm{H}_{2} \mathrm{O}\right)_{2}$ [GGP]. In all cases, the researchers found that stable Pt dimers already formed after a single reduction step of a system containing a free $\mathrm{Pt}(\mathrm{II})$ complex and a $\mathrm{Pt}(\mathrm{II})$ complex covalently bound to DNA. As a basic result, they observed that the presence of nucleotide ligands results in the formation of Pt dimers with bonds that are stronger than dimers formed homogeneously. Furthermore, they hypothesized that the catalytic role of DNA may extend to future reduction-addition steps of cluster growth. The researchers inferred from calculated electron affinities that the metal dimers formed at DNA are expected to be preferred sites for further reductions, leading to easier incorporation of $\mathrm{Pt}$ atoms from solution.

Experimentally, DNA is "activated" by incubation in an aged $\mathrm{K}_{2} \mathrm{PtCl}_{4}$ solution. All possible metal-binding sites along the DNA become occupied when high complex-tonucleotide ratios and long activation times, $t_{\mathrm{a}}$, are employed. Dimethylamine borane is added after activation to reduce $\mathrm{Pt}$ (II) to metallic platinum. UV-visible 
spectroscopy was used to monitor the reduction kinetics. Transmission electron microscopy (TEM) and scanning force microscopy were used to analyze the products. Regular, continuous chains of nanoparticles, of 4-nm average diameter, form over the contour length of DNA in contrast to the large cluster agglomerates, of $\sim 50$-nm average diameter, that form in the absence of DNA. High-resolution TEM images of the nanoparticles show latticeplane distances identical to bulk platinum.

The researchers showed that the metallization rate and the balance between heterogeneous and homogeneous nucleation can be controlled by varying $t_{\mathrm{a}}$. An accelerated process and altered nucleation behavior results from increasing $t_{\mathrm{a}}$. Vanishingly small activation times result in mostly homogeneously nucleated large clusters and a small number of small clusters on the DNA strands. Exclusively heterogeneous nucleation takes place after long activation times $(\sim 20 \mathrm{~h})$. The researchers expect that heterogeneous cluster nucleation may be influenced by the DNA composition because it has been previously shown that the complexation kinetics is nucleotide-specific to a certain degree.

Mertig suggests that "strong donor ligands other than DNA, bound to metal ions before (or during) the reduction process to form organometallic complexes, may induce cluster nucleation in a similar way." Mertig expects formation of similarly nucleated platinum nanoparticles on proteins if heterocyclic amino acids are present.

STEVEN TROHALAKI

\section{Thin Oxide Films Crystallized with Low-Temperature Anneals}

Sangmoon Park and colleagues from Oregon State University, along with collaborators from ReyTech Corp. and Hewlett-Packard, reported their work on low-temperature thin-film deposition and crystallization in the July 5 issue of Science. Park and colleagues described their deposition and annealing techniques, which allowed for the creation of thin-film crystalline oxides for applications in displays, electronics, and energy storage. Typically, a high-temperature processing step is required to crystallize deposited amorphous films. This creates constraints to obtain desirable characteristics of the film while maintaining stability of the substrate, in addition to increasing the cost. By using a unique method of preparing the oxide powders by precipitation and hydrothermal dehydration, and depositing them using the successive ionic layer adsorption and reaction (SILAR) deposition method, the research- ers have created amorphous films that crystallize under low-temperature hydrothermal annealing.

The oxide powders were created by dehydrating a hydroxo precipitate under hydrothermal conditions to form an anhydrous crystalline oxide. The SILAR process consists of repeated monolayer coatings of material and water rinsing until the desired film thickness is achieved. The samples underwent 700 cycles of $\sim 0.1 \mathrm{M}$ solutions and rinse baths for $10 \mathrm{~s}$ each by robotic control to obtain layer thicknesses of $\sim 250 \mathrm{~nm}$. The sam- ples were then dehydrated for $\sim 12 \mathrm{~h}$. Films of several oxides, $\left(\mathrm{Zn}_{2} \mathrm{SiO}_{4}, \mathrm{ZrO}_{2}\right)$ and $\left(\mathrm{MnO}_{2}, \mathrm{Mn}_{2} \mathrm{O}_{3}\right)$, were deposited on $\mathrm{Si}_{3} \mathrm{~N}_{4} / \mathrm{Si}$ and $\mathrm{SiO}_{2} / \mathrm{Si}$ substrates, respectively, using the SILAR process, creating amorphous films. Low temperature (378-473 K) hydrothermal annealing in a sealed 23-ml Teflon-lined Parr reactor with $0.15 \mathrm{ml}$ of water produced highly crystalline films, as determined by x-ray powder diffraction. Annealing the asdeposited films at higher temperatures (773-923 K) did not produce crystalline material in the case of $\mathrm{Zn}_{2} \mathrm{SiO}_{4}$, and pro-

\section{CVD-XVI/EUROCVD-14 April 27-May 2, 2003 \\ Held in conjunction with the 203rd Meeting of the Electrochemical Society \\ CVD-XVI/EUROCVD-14 continues the tradition of CVD symposia sponsored by the Electrochemical Society since 1970 and the European CVD conferences launched in Paris in 1977. It will provide an opportunity for scientists to present and review research on both fundamental and applied topics, exchange ideas, and promote international cooperation. \\ Abstract Deadline: September 13, 2002 \\ Send submission of titles and short abstracts ( 100 words) to: Francis Teyssandier, Institute of Materials Science and Process Engineering CNRS-UPR8521 \\ For complete details on this event, visit www.electrochem.org Rambla de la Thermodynamique F-66100 Perpignan Cedex, FRANCE e-mail teyssandier@univ-perp.fr fax 33-4-68-55-68-69

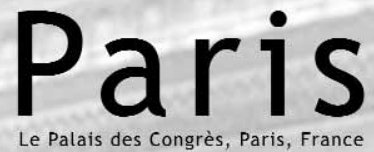

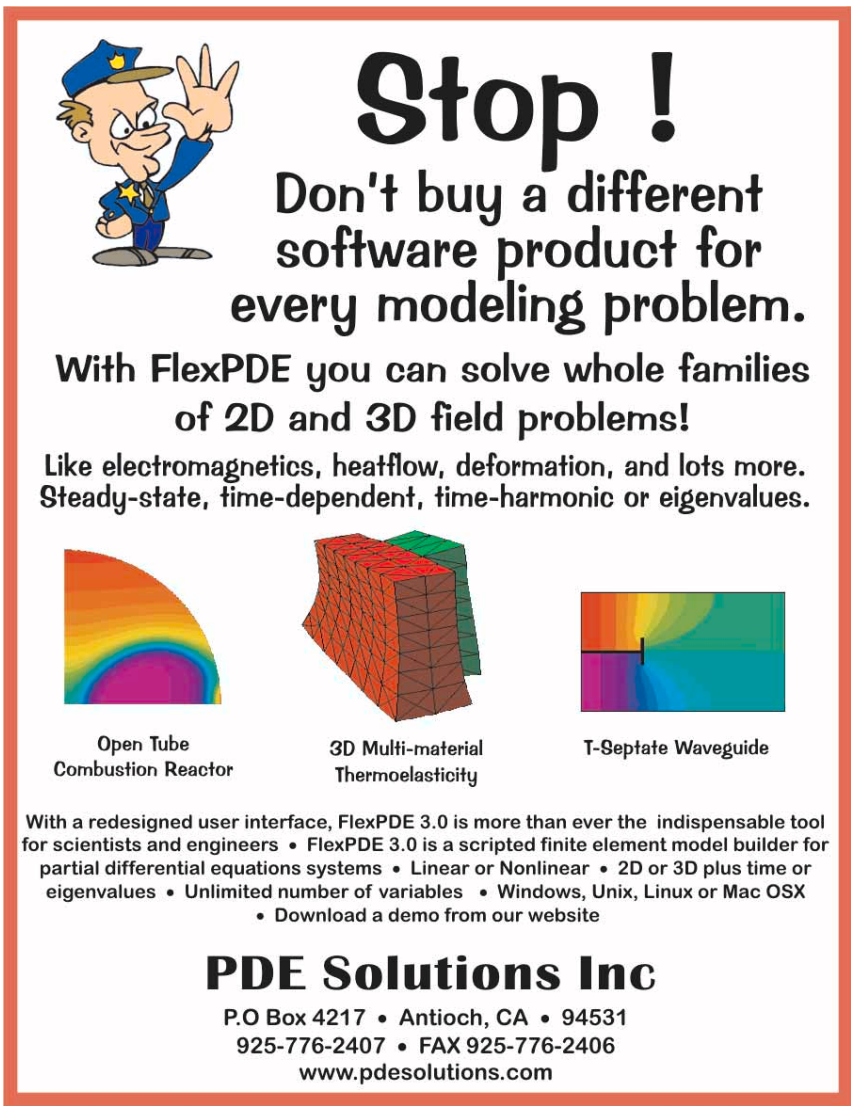

Circle No. 10 on Inside Back Cover 